\title{
Dynamic Mobility-Aware Interference Avoidance for Aerial Base Stations in Cognitive Radio Networks
}

\author{
Ali Rahmati*, Xiaofan $\mathrm{He}^{\dagger}$, Ismail Guvenc*, and Huaiyu Dai* \\ ${ }^{*}$ Department of Electrical and Computer Engineering, NC State University, Raleigh, NC, US \\ ${ }^{\dagger}$ School of Electronic Information, Wuhan University, Wuhan, China \\ Email: \{arahmat@ncsu.edu, xiaofanhe@whu.edu.cn, iguvenc@ncsu.edu, hdai@ncsu.edu\}
}

\begin{abstract}
Aerial base station (ABS) is a promising solution for public safety as it can be deployed in coexistence with cellular networks to form a temporary communication network. However, the interference from the primary cellular network may severely degrade the performance of an ABS network. With this consideration, an adaptive dynamic interference avoidance scheme is proposed in this work for ABSs coexisting with a primary network. In the proposed scheme, the mobile ABSs can reconfigure their locations to mitigate the interference from the primary network, so as to better relay the data from the designated source(s) to destination(s). To this end, the single/multi-commodity maximum flow problems are formulated and the weighted Cheeger constant is adopted as a criterion to improve the maximum flow of the ABS network. In addition, a distributed algorithm is proposed to compute the optimal ABS moving directions. Moreover, the trade-off between the maximum flow and the shortest path trajectories is investigated and an energy-efficient approach is developed as well. Simulation results show that the proposed approach is effective in improving the maximum network flow and the energy-efficient approach can save up to $39 \%$ of the energy for the ABSs with marginal degradation in the maximum network flow.
\end{abstract}

Index Terms-Aerial base station, UAV, optimal trajectory, maximum flow problem, energy efficiency.

\section{INTRODUCTION}

Recently, aerial base stations (ABSs) have been introduced as an effective solution for a variety of important applications, including environmental monitoring, public safety, disaster relief, search/rescue, surveillance, and purchase delivery [1], [2]. The federal aviation administration (FAA) reported that the number of ABSs will exceed 7 million in 2020 [3]. The ABSs can be implemented using unmanned aerial vehicles (UAVs). Being cost-effective, flexible and suitable for low-latency communication in 5G [4], [5], the ABSs are considered as a promising supplement to conventional terrestrial base stations to provide broadband connectivity in complex scenarios.

Nonetheless, forming an ABS communication network for the aforementioned applications is highly non-trivial. Particularly, since there is no regulation and pre-allocated spectrum band for the ABSs [6], [7], in most scenarios the ABS network may need to share the spectrum with an existing primary network. Without careful management, the performance of both the ABS network and the primary network may be considerably degraded due to mutual interference. This clearly suggests a

This work was supported in part by the National Science Foundation under grants ECCS-1444009 and CNS-1824518. compelling need for developing innovative ABS positioning and mobility management schemes.

With this consideration, the interference avoidance problem for the ABS network formation is studied in this work, with the idea that the mobility feature of ABSs offers a built-in advantage for dynamic re-positioning and thus interference avoidance. Specifically, a novel dynamic mobility-aware interference avoidance scheme is proposed in this work by leveraging spectral graph theory and incorporating practical ABS channel models. Through the proposed scheme, the ABSs can dynamically reconfigure their locations to avoid the interference caused by the primary network and optimize the data flow from the designated source(s) to destination(s).

The contributions of this work can be summarized as follows:

- ABS network interference avoidance is formulated as a single/multi-commodity maximum flow problem.

- Taking advantage of the inherent mobility feature of the ABSs and incorporating practical ABS channel model, an adaptive interference avoidance scheme is developed. Using the proposed scheme, the ABSs can effectively reconfigure their positions to avoid the interference and improve the data flow from the source(s) to the destination(s).

- The weighted Cheeger constant for 3D scenarios is introduced as an effective metric to improve the ABS network connectivity.

- A distributed algorithm for computing the optimal ABS moving directions is presented.

- The trade-off between energy efficiency and the maximum flow of the ABS network is investigated.

The rest of the paper is organized as follows. In Section III the related works are discussed. In Section IIII, the system model is presented. In Section IV] the single-commodity maximum flow problem is formulated and the weighted Cheeger constant is adapted to 3D coordinates. The multi-commodity problem is presented in Section V] In Section VI, several practical considerations related to ABS deployment are discussed. Section VII investigates the performance and effectiveness of the proposed scheme via extensive simulations, and finally, Section VIII concludes the paper.

\section{RELATED WORKS}

Due to the lack of regulation and spectrum scarcity, the ABSs may have to coexist with a primary network in practice. 
Thus, ABS network management is necessary to mitigate the interference from/to the primary network. In [8], the energy efficiency of a cognitive radio ABS is investigated in the presence of primary transceivers. In [9], a dynamic transmission duty cycle allocation scheme is proposed for coexisting ABS and cellular/WiFi networks based on regret game. In addition, it was shown that the duty cycle allocation strategy based on the correlated equilibrium of the game outperforms static duty cycle allocation. In spite of these pioneering works, the dynamic ABS positioning problem in the cognitive radio setting has not been considered, where the key research problem is how to design the trajectories of the ABSs to mitigate the mutual interference between the ABS network and the primary network.

The ideas of mobility-aware interference-avoidance, path planning and trajectory optimization have already been explored for ABS networks in the literature [10]-[15]. However, mobilityaware interference-management becomes more challenging in the cognitive radio setting and so is the design of effective interference avoidance trajectories for the ABS. The authors in [10] proposed energy-efficient ABS trajectory optimization for given ABS initial and final locations, and showed that this scheme outperforms other existing schemes, including those based on heuristic rate-maximization and energy-minimization, in terms of ABS communications. In contrast, the final locations of the ABSs in our work are assumed unfixed and derived towards the maximum flow metric. In [12], the effectiveness of ABS deployment in overload and outage situations is investigated where the relay placement, the number of relays, and the relay transmit power are analyzed. In [14]-[17], the connectivity of the ABS network is characterized by its Fiedler value and algebraic connectivity. However, their approach is different from ours, as they consider neither the cognitive radio setting nor dynamic interference avoidance.

In [18], the importance of algebraic connectivity for controlling the positions of ABSs towards a desired formation is shown. A mixed-integer linear programming is formulated in order to maximize the algebraic connectivity and its upper bounds are obtained based on cutting plane methods. In [19], ABS-based monitoring applications are considered, where ABSs are deployed to relay $[20]-[22]$ and transmit time information between the vehicles and the control stations. The key difference between [18], [19] and our work is that we consider mobile ABSs to dynamically improve the network connectivity in a cognitive radio setting.

\section{SySTEM MODEL}

\section{A. Scenario Description}

Consider a scenario in which a set of sources of interference (SIs) (e.g., base stations, small cells, or user equipment in the primary network [23], [24]) and ABSs coexist in the network. The ABSs aim to form a temporary network to relay data from a designated terrestrial source to a terrestrial destination. To mitigate the interference from the SIs, the ABS nodes can change their geographic locations in the $3 \mathrm{D}$ space.
We assume that the SIs can be detected using existing sensing methods as in [25].

Assume that there is a set $\mathcal{N}$ of $N$ nodes, including the ABSs, the terrestrial source $(s)$, and the destination $(d)$ in the system, in addition to a set $\mathcal{M}$ of $M$ SIs. The positions of the ABSs and the SIs are denoted by: $\left\{r_{i}=\left(x_{i}, y_{i}, h_{i}\right) \in \mathbb{R}^{3}, i=1, \ldots, N\right\}$ and $\left\{r_{p}=\left(x_{p}, y_{p}\right) \in \mathbb{R}^{2}, p=1, \ldots, M\right\}$, respectively. Note that the third coordinates of the terrestrial source and destination are equal to zero. In the considered model, the ABS network consists of the source, the destination, and the ABSs.

Matrix $\mathbf{A}=\left[a_{i, j}\right]_{i, j=1}^{N}$ is the so-called generalized adjacency matrix of the ABS network [26], in which the elements $a_{i, j}$ 's are defined based on the 1-nat information exchanging time between nodes $i$ and $j$ as:

$$
a_{i, j}=\left\{\begin{array}{ll}
B\left(\frac{1}{\ln \left(1+\operatorname{SIR}_{i, j}\right)}+\frac{1}{\ln \left(1+\operatorname{SIR}_{j, i}\right)}\right)^{-1}, & i \neq j \\
0, & i=j
\end{array},\right.
$$

where $B$ is the total bandwidth of the system, and $\operatorname{SIR}_{i, j}$ is the signal-to-interference ratio at node $j$ for the transmission from node $i$ assuming unit transmission power from the transmitter and interferers. In this work, $\operatorname{SIR}_{i, j}$ is given by:

$$
\operatorname{SIR}_{i, j}=\frac{g_{i, j}}{\sum_{p=1}^{M} g_{p, j}+\sum_{k \in \mathcal{Q}_{i, j}} u\left(d_{j, k} / r_{\text {int }}\right)},
$$

where $g_{i, j}$ stands for the average channel gain between node $i$ and node $j$, further discussed below; $\mathcal{Q}_{i, j}$ denotes the set of all the nodes excluding node $i$ and $j$, and $d_{i, j}$ is the Euclidean distance between node $i$ and $j$. The smoothed step function is defined as:

$$
u(y)=\zeta \frac{\exp \left(-\kappa y-\log y_{0}\right)}{1+\exp \left(-\kappa y-\log y_{0}\right)},
$$

where $y_{0}$ is an arbitrarily small positive number and $\zeta$ and $\kappa$ are design parameters. These parameters together with $r_{\text {int }}$ determine the required safety separation of the ABSs in practice. The first term of the denominator in (2) is related to the interference caused by the SIs, while the second term is included to prevent the ABSs from getting too close to and colliding with each others.

Let us define the matrix $\mathbf{D}=\operatorname{diag}\left\{\beta_{1}, \ldots, \beta_{N}\right\}$ as the generalized degree matrix of the ABS network with $\beta_{i}=$ $\sum_{\{j \mid j \neq i\}} a_{i, j}$. Thus, the Laplacian matrix of the network graph is defined as $\mathbf{L}=\mathbf{D}-\mathbf{A}$. Since both terrestrial and aerial nodes are involved, it is necessary to capture the corresponding channel models. In the following, the air to air (A2A) and air to ground $(\mathrm{A} 2 \mathrm{G})$ channel models for $\mathrm{ABS}$ nodes are presented in details.

\section{B. $A B S A 2 A$ and $A 2 G$ Channel Models}

Denote the set of the aerial nodes by $\mathcal{A}=\mathcal{N} \backslash\{s, d\}$, and the set of terrestrial nodes by $\mathcal{G}=\mathcal{M} \cup\{s, d\}$. The wireless links between node $i \in \mathcal{A}$ (i.e., ABSs) and node $j \in \mathcal{G}$ (i.e., the source, the destination, and the interference sources) can either be line-of-sight (LoS) or non-line-of-sight (NLoS). However, only LoS channels are considered for the links 
between the nodes $i, j \in \mathcal{A}$ [27]. In our model, we assume channel reciprocity for all links.

The signal propagation in A2G channel is mainly affected by the obstacles and buildings in the environment. Specifically, an A2G communication link can be either LoS or NLoS with a certain probability depending on the location, the height, the number of obstacles, as well as the elevation angle between the ABS and the ground node. In our scheme, we assume a widely-used probabilistic path-loss model provided by the International Telecommunication Union (ITU). In particular, the path-loss between an ABS node $i \in \mathcal{A}$ and a ground node $j \in \mathcal{G}$ is given by [28], [29]:

$$
\Gamma_{i, j}= \begin{cases}\left(K_{o} d_{i, j}\right)^{\alpha} \mu_{\mathrm{LoS}}, & \text { if LoS link } \\ \left(K_{o} d_{i, j}\right)^{\alpha} \mu_{\mathrm{NLoS}}, & \text { if NLoS link, }\end{cases}
$$

where $\mu_{\mathrm{LoS}}$ and $\mu_{\mathrm{NLOS}}$ are different attenuation factors for the LoS and the NLoS links, respectively, $f_{c}$ is the carrier frequency, $\alpha$ is the path-loss exponent, and $c$ is the speed of light, and $K_{o}=\frac{4 \pi f_{c}}{c}$. For aerial nodes $i \in \mathcal{A}$ and the ground node $j \in \mathcal{G}$, the probability of having an LoS link is given by [28], [29]:

$$
P_{i, j}^{\mathrm{LoS}}=\frac{1}{1+\psi \exp \left(-\eta\left[\theta_{i j}-\psi\right]\right)},
$$

where $\psi$ and $\eta$ are constants depending the carrier frequency and the conditions of the environment. The elevation angle between an ABS node $i \in \mathcal{A}$ and a ground node $j \in \mathcal{G}$ is given by:

$$
\theta_{i, j}=\frac{180}{\pi} \times \sin ^{-1}\left(\frac{\Delta h_{i, j}}{d_{i, j}}\right),
$$

where $\Delta h_{i, j}$ is the height difference between node $i$ and node $j$. Clearly, the probability of NLoS is $P_{i, j}^{\mathrm{NLoS}}=1-P_{i, j}^{\mathrm{LoS}}$. The average path-loss of the $\mathrm{A} 2 \mathrm{G}$ link from node $i \in \mathcal{A}$ to node $j \in \mathcal{G}$ can be obtained as [28]:

$$
\bar{L}_{i, j}^{\mathrm{A} 2 \mathrm{G}}=\left(K_{o} d_{i, j}\right)^{\alpha}\left[P_{i, j}^{\mathrm{LoS}} \times \mu_{\mathrm{LoS}}+P_{i, j}^{\mathrm{NLoS}} \times \mu_{\mathrm{NLoS}}\right] .
$$

As we assume LoS links between different ABSs, the pathloss model between ABS $i$ and ABS $j$ is given by [28]:

$$
L_{i, j}^{\mathrm{A} 2 \mathrm{~A}}=\left(K_{o} d_{i, j}\right)^{\alpha} \mu_{\mathrm{LoS}} .
$$

Based on this, the channel power gain between an ABS $i$ and a ground/aerial node $j$ is given by:

$$
g_{i j}= \begin{cases}\frac{1}{L_{i, j}^{\mathrm{A} 2 \mathrm{~A}}}, & \mathrm{~A} 2 \mathrm{~A} \text { link } \\ \frac{1}{\bar{L}_{i j}^{\mathrm{A} \mathrm{G}}}, & \mathrm{A} 2 \mathrm{G} \text { link. }\end{cases}
$$

With the path-loss models for the A2A and A2G links in the system and the network topology, one can obtain the generalized Laplacian matrix of the network. In the next section, we formulate the single-commodity maximum flow problem for the considered scenario.

\section{SINGLE-COMMODITY MAXIMUM FLOW PROBLEM FOR ABS NETWORK FORMATION}

In this section, the ABS network formation problem for the case of a single pair of source and destination is formulated as a single-commodity maximum flow problem. In this problem, the flow through each edge is upper bounded by the capacity of the corresponding wireless link. The incoming and outgoing flows for each ABS are assumed equal (i.e., balanced flows).

\section{A. The Single-commodity Maximum Flow Problem}

Define a directed flow graph $G=(\mathcal{N}, \mathcal{E})$ where each edge has the capacity $a_{i, j}$, and $\mathcal{E}$ denotes the set of available edges in the network. In the single-commodity maximum flow problem, the task is to determine the maximum amount of flow that can be sent from the source to the destination.

For each link $(i, j) \in \mathcal{E}$, let $f_{i, j}$ denote the flow on link $(i, j)$, which admits $0 \leq f_{i, j} \leq a_{i, j}$. Mathematically, the singlecommodity maximum flow problem can be defined as:

$$
\begin{array}{ll}
\max & \sum_{j:(s, j) \in \mathcal{E}} f_{s, j} \\
\text { s.t } & \sum_{\{i:(i, j) \in \mathcal{E}\}} f_{i, j}-\sum_{\{l:(j, l) \in \mathcal{E}\}} f_{j, l}=0, \quad \forall j \in \mathcal{N} \backslash\{s, d\}, \\
& 0 \leq f_{i, j} \leq a_{i, j}, \forall(i, j) \in \mathcal{E} .
\end{array}
$$

The first constraint corresponds to the assumption of balanced flows for all the nodes, except for the source and the destination. The well-known Ford-Fulkerson algorithm [30] can be employed to solve the above maximum flow problem.

\section{B. Cheeger Constant}

For the normalized Laplacian matrix $\mathcal{L}=\mathbf{D}^{-1 / 2} \mathbf{L} \mathbf{D}^{-1 / 2}$, the Cheeger constant is defined as [26]:

$$
h(\mathcal{L})=\min _{S} \frac{\sum_{i \in S, j \in \bar{S}} a_{i, j}}{\min \{\operatorname{vol}(S), \operatorname{vol}(\bar{S})\}},
$$

where $S \subset \mathcal{N}$ is a subset of the nodes and $\operatorname{vol}(S)=\sum_{i \in S} \beta_{i}$. The well-known normalized Cheeger's inequality is given by [31]:

$$
\lambda_{2}(\mathcal{L}) / 2 \leq h(\mathcal{L}) \leq \sqrt{2 \lambda_{2}(\mathcal{L})} .
$$

In (7), the second smallest eigenvalue of the network Laplacian matrix $\lambda_{2}(\mathcal{L})$ is a measure of the connectivity of the graph, defined as [31]:

$$
\lambda_{2}(\mathcal{L})=\min _{v \neq \mathbf{0}, v \perp \mathbf{1}} \frac{\langle\mathcal{L} v, v\rangle}{\langle v, v\rangle},
$$

where 0 (1) is the vector with all coordinates equal to 0 (1). Based on (7), in order to maximize the Cheeger constant (6), one can maximize the second smallest eigenvalue of the network (also known as the algebraic connectivity). However, one drawback of $h(\mathcal{L})$ is that it is invariant to the scaling of the link capacities $a_{i, j}$, while the maximum flow of the network for a given source-destination pair is obviously not. To address this, we utilize the weighted version of the Cheeger constant [32].

\section{Weighted Cheeger Constant}

Besides the scaling issue, the regular Cheeger constant also blindly aims at improving the weakest links in the network and may fail to emphasize the flow for a given source-destination 
pair. Thus, we need to distinguish the source and destination nodes from the ABSs nodes, and weighted Cheeger constant comes as a remedy, defined as [32]:

$$
h_{\mathbf{W}}(\mathcal{L})=\min _{S} \frac{\sum_{i \in S, j \in \bar{S}} a_{i, j}}{\left.\min \left\{|S|_{\mathbf{W}}\right),\left(|\bar{S}|_{\mathbf{W}}\right)\right\}},
$$

where the weighted cardinality $|S|_{\mathbf{W}}=\sum_{i \in S} w_{i}$, and $w_{i} \geq 0$ is the weight of the node $i$, adopted to stress the bottleneck for the flow between the source and destination nodes. The weighted Laplacian matrix is defined as:

$$
\mathcal{L}_{\mathbf{W}}=\mathbf{W}^{-1 / 2} \mathcal{L} \mathbf{W}^{-1 / 2}, \text { with } \mathbf{W}=\operatorname{diag}\left\{w_{1}, \ldots, w_{n}\right\} .
$$

To achieve our goal, the ideal choice is to consider the weights for the source and destination as 1 , and those for the ABSs as 0 . However, this makes the problem intractable because the weight matrix $\mathbf{W}$ will be non-invertible. As a result, a practical setting is to choose the weights large for the source(s) and destination(s) and small enough for the ABSs. Similar to (8), the weighted second smallest eigenvalue $\lambda_{2}\left(\mathcal{L}_{\mathbf{W}}\right)$ can be defined as:

$$
\lambda_{2}\left(\mathcal{L}_{\mathbf{W}}\right)=\min _{v \neq \mathbf{0}, v \perp \mathbf{W}^{1 / 2} \mathbf{1}} \frac{\left\langle\mathcal{L}_{\mathbf{W}} v, v\right\rangle}{\langle v, v\rangle} .
$$

It has been shown in [32] that the following weighted Cheeger's inequalities hold:

$$
\lambda_{2}\left(\mathcal{L}_{\mathbf{W}}\right) / 2 \leq h_{\mathbf{W}}\left(\mathcal{L}_{\mathbf{W}}\right) \leq \sqrt{2 \delta_{\max } \lambda_{2}\left(\mathcal{L}_{\mathbf{W}}\right) / w_{\text {min }}}
$$

where $\delta_{\max }$ is the maximum node degree, and $w_{\min }=\min _{i} w_{i}$. By maximizing $\lambda_{2}\left(\mathcal{L}_{\mathrm{W}}\right)$, the lower bound of the weighted Cheeger constant increases. Hence, the ABS nodes can change their locations in order to maximize $\lambda_{2}\left(\mathcal{L}_{\mathbf{W}}\right)$ and thus $h_{\mathbf{W}}(\mathcal{L})$.

\section{ABSs Moving Directions Towards Maximum Flow}

In order to maximize the weighted algebraic connectivity $\lambda_{2}\left(\mathcal{L}_{\mathbf{W}}\right)$, each ABS can move along the spatial gradient of $\lambda_{2}\left(\mathcal{L}_{\mathrm{W}}\right)$. Given the instantaneous position of an ABS node $i$, its spatial gradient along $x$-axis can be obtained as follows:

$$
\begin{aligned}
\frac{\partial \lambda_{2}\left(\mathcal{L}_{\mathbf{W}}\right)}{\partial x_{i}} & =\mathbf{x}^{f^{T}} \frac{\partial\left(\mathcal{L}_{\mathbf{W}}\right)}{\partial x_{i}} \mathbf{x}^{f} \\
& =\sum_{\{p, q: p \sim q\}}\left[\frac{x_{p}^{f}}{\sqrt{w_{p}}}-\frac{x_{q}^{f}}{\sqrt{w_{q}}}\right]^{2} \frac{\partial a_{p, q}}{\partial x_{i}},
\end{aligned}
$$

where $\mathbf{x}^{f}$ is the Fiedler vector (i.e., the eigenvector corresponding to the second smallest eigenvalue $), x_{p}^{f}\left(\mathbf{x}_{q}^{f}\right)$ is the $p^{\text {th }}\left(q^{\text {th }}\right)$ component of $\mathbf{x}^{f}$, and $p \sim q$ means that nodes $p$ and $q$ are connected. In order to compute (13), we need to compute $\frac{\partial a_{p, q}}{\partial x_{i}}$, and it should be noted that if $p=q, \frac{\partial a_{p, q}}{\partial x_{i}}=0$. Specifically:

$$
\begin{aligned}
\frac{\partial a_{p, q}}{\partial x_{i}} & =B\left(\frac{1}{\ln \left(1+\operatorname{SIR}_{p, q}\right)+\ln \left(1+\operatorname{SIR}_{q, p}\right)}\right)^{-2} \\
& \times\left[\frac{1}{\left(1+\operatorname{SIR}_{p, q}\right) \ln ^{2}\left(1+\operatorname{SIR}_{p, q}\right)} \frac{\partial \operatorname{SIR}_{p, q}}{\partial x_{i}}\right. \\
& \left.+\frac{1}{\left(1+\operatorname{SIR}_{q, p}\right) \ln ^{2}\left(1+\operatorname{SIR}_{q, p}\right)} \frac{\partial \operatorname{SIR}_{q, p}}{\partial x_{i}}\right] .
\end{aligned}
$$

For coordinates $y$ and $z$, the moving directions can be obtained similarly.

\section{Multi-Commodity Maximum Flow Problem}

In this section, the ABS interference-avoidance problem is investigated under both the multicast and the multi-unicast scenarios [33]. The maximum concurrent flow is defined as maximum of $f^{m}$ so that $f^{m} D(k)$ units from commodity $1 \leq$ $k \leq K$ can be transferred to the destination at the same time in which $D(k)$ is the demand for the commodity $k$. In the multi-commodity problem, typically there are more than one destination in the network [33]. In the multicast scenario, the network conducts the flow from the source to multiple destinations, while in the multi-unicast scenario, there are multiple pairs of source-destination with individual flows for each pair. The minimum multicut $C^{m}$ for the multi-commodity problem is defined as [33]:

$$
C^{m}=\min _{S} \frac{\sum_{i \in S, j \in \bar{S}} a_{i, j}}{\sum_{k \in \mathcal{K}(S)} D(k)},
$$

where $\mathcal{K}(S)=\left\{k: v_{s_{k}} \in S\right.$ and $v_{d_{k}} \in \bar{S}$, or $v_{s_{k}} \in$ $\bar{S}$ and $\left.v_{d_{k}} \in S\right\}$, and $v_{s_{k}}$ and $v_{d_{k}}$ are the source and the destination for commodity $k$, respectively. Similar to the approach presented in Section IV-C by defining $\mathbf{W}^{(k)}=$ $\operatorname{diag}\left\{w_{1}^{(k)}, \ldots, w_{n}^{(k)}\right\}$, the multi-weighted Cheeger constant [32] can be defined as:

$$
h_{\mathbf{W}}^{(K)}(\mathcal{L})=\min _{S} \frac{\sum_{i \in S, j \in \bar{S}} a_{i, j}}{\left.\sum_{k=1}^{K} \gamma_{k} \min \left\{|S|_{\mathbf{W}^{(k)}}\right),\left(|\bar{S}|_{\mathbf{W}^{(k)}}\right)\right\}},
$$

where $\left.\gamma_{k}=\min ^{-p}\left\{|S|_{\mathbf{W}^{(k)}}\right),\left(|\bar{S}|_{\mathbf{W}^{(k)}}\right)\right\}$ for commodity $k=1, \ldots, K$. Parameter $\gamma_{k}$ is inversely proportional to the $k^{\text {th }}$ flow bottleneck. The value of $p$ is related to the weight $\mathbf{W}^{(k)}$ and its most suitable value can be obtained via numerical explorations. Defining $\mathbf{\mathbf { W }}=\operatorname{diag}\left\{\bar{w}_{1}, \ldots, \bar{w}_{n}\right\}$ where $\bar{w}_{i}=\sum_{k=1}^{K}\left(w_{i}^{(k)}\right)^{1-p}$, the weighted Laplacian matrix is given by $\mathcal{L}_{\overline{\mathbf{W}}}^{(K)}=\overline{\mathbf{W}}^{-1 / 2} \mathcal{L} \overline{\mathbf{W}}^{-1 / 2}$. As in Section IV-D the ABSs can take $\lambda_{2}\left(\mathcal{L}_{\overline{\mathbf{W}}}^{(K)}\right)$ as a metric to reconfigure their positions, so as to achieve a better performance as compared to the case of using $\lambda_{2}(\mathcal{L})$.

\section{PRACTICAL CONSIDERATIONS}

\section{A. ABSs with Limited Communication Range}

The discussions in the previous sections assume that the network graph is a complete graph, which may not be true in some scenarios due to the limited communication range $R_{i}^{\text {th }}$ of the ABSs. The value of $R_{i}^{\text {th }}$ depends on the transmission power of ABS $i$. To capture this effect, it is assumed in this subsection that, for two given ABSs $i$ and $j$, a communication link (or equivalently, an edge) exists only if $d_{i, j} \leq R_{i}^{\text {th }}$. Hence, the definition of the adjacency matrix $\mathbf{A}$ of the ABS network graph is modified accordingly as

$$
a_{i, j}=\left\{\begin{array}{cc}
c_{i, j}, & \text { if } i \neq j \text { and } d_{i, j} \leq R_{i}^{t h} \\
0, & \text { otherwise }
\end{array}\right.
$$

where $c_{i, j}$ is defined as in (1). 


\section{B. Distributed Algorithm for Computing ABS Directions}

As can be seen from (13), to compute the moving directions, each ABS $i$ needs the Fiedler vector $\mathbf{x}^{f}$ of the Laplacian matrix. To compute the Fiedler vector in a centralized way, each ABS needs to know the whole matrix $\mathcal{L}_{\mathrm{W}}$ at each time-slot which is inefficient both in terms of communication overhead and computation, especially when the number of ABSs is large. However, having a closer look at (13), it can be seen that ABS $i$ does not need all the elements of $\mathbf{x}^{f}$ to compute its moving direction. It just needs the elements of $\mathbf{x}^{f}$ corresponding to its neighbors. As a result, a distributed algorithm can be used to compute the Fiedler vector to reduce both the communication and the computation complexity for the ABS nodes.

Based on the algorithm proposed in [34], the Fiedler vector can be computed in a distributed fashion. During each iteration, the nodes are assumed to perform a pre-defined task and share the result with their neighbors. When the algorithm is done, each node eventually has access to its corresponding entry in the Fiedler vector. By increasing the number of iterations, the error of the computed Fiedler vector is decreased. It is worth mentioning that to compute the moving direction for the ABS $i$, the elements of the Fiedler vector of its neighbors are needed. Here, we assume that the ABSs can exchange such information with their neighbors. Algorithm 1 describes the details of the proposed distributed algorithm based on [34].

\section{Energy Consumption and Maximum Flow Trade-off}

Since the ABSs are using batteries as their energy sources, developing energy efficient trajectory is critical. However, the optimal trajectories for maximum flow of the network are not necessarily the best trajectories for energy saving. If the ABSs compute their moving directions solely using the maximum flow criterion in each iteration, the trajectory between the initial and the final points is usually not the most energy-efficient. In some applications, the ABSs can move in a more energyefficient way at the expense of a longer operation time.

To achieve the desired energy-efficient mobility management for the ABSs, suppose that each time-slot $t$ consists of $t^{\mathrm{c}}$ (the computation time) and $t^{\mathrm{m}}$ (the moving time). The computation time is the amount of time needed to compute the moving directions as in (13) which, for ABS $i$, can be obtained as $t_{i}^{\mathrm{c}}=\frac{K_{i}^{2}}{e_{i}}[11]$; here, $K_{i}$ is the amount of data that must be processed before movement and $e_{i}$ is the processing speed of ABS $i$. The moving time is the amount of time needed for each $\mathrm{ABS}$ to traverse the trajectory which is equal to $t_{i}^{\mathrm{m}}=\frac{D_{i}}{v_{i}}$ [35]; here, $D_{i}$ is the length of the path traversed by $\mathrm{ABS} i$ and $v_{i}$ is the ABS speed. Let us assume that the ABSs try to compute their final locations in $L$ iterations (time slots) according to the maximum flow metric. Having the final location using maximum-flow criterion, the ABSs can employ an energy efficient trajectory to reach their final locations. This imposes $L t_{i}^{\mathrm{c}}$ latency to the network before they start to move as compared to the maximum flow trajectory.

More specifically, denote the initial and the final locations of the ABS $i$ obtained using the method in Section IV-C by $q_{i}[0]$, and $q_{i}[L]$, respectively. Here, we assume the ABSs can

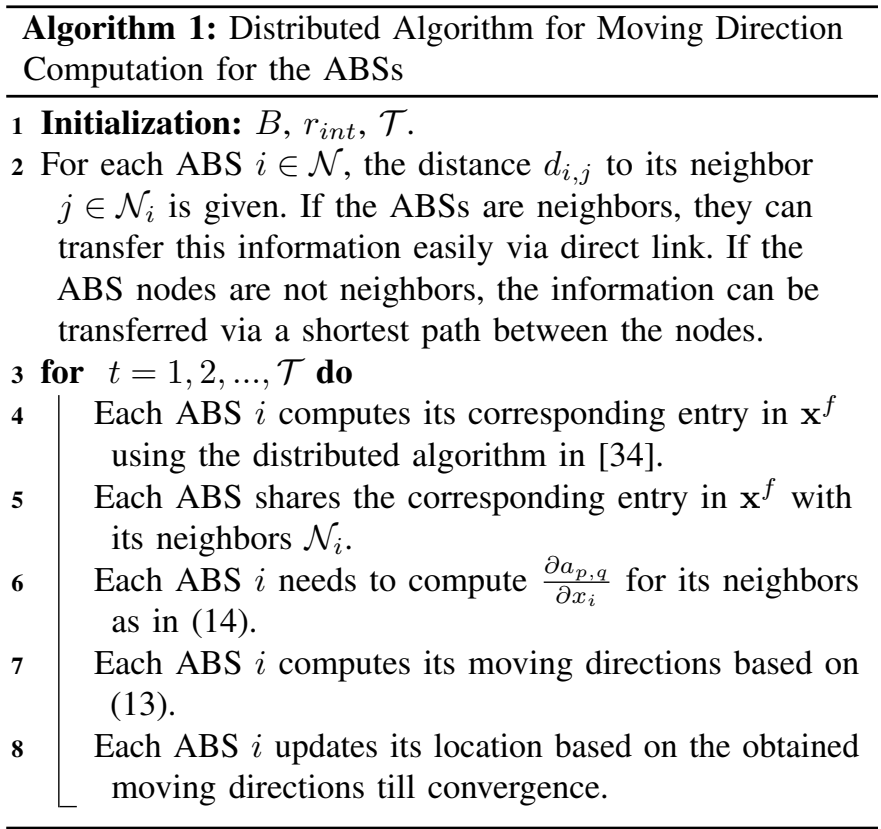

converge to the network maximum flow in $L$ iterations using the weighted Cheeger constant approach. The ABSs can wait $L t_{i}^{\mathrm{c}}$ amount of time to compute $q_{i}[L]$ and then choose the most energy efficient trajectory to traverse to the final location. As a result, the energy efficient approach imposes $L t_{i}^{\mathrm{c}}$ amount of computation delay to the network before the ABSs start to move. After reaching the final location, ABSs can relay the information with the maximum flow.

Assuming rotary wing ABSs, the energy consumption of each ABS $i$ between two stopping positions with path length $D_{i}$ can be computed as [35]:

$$
E_{i}=\frac{D_{i}}{v_{i}}\left(P_{i}^{\mathrm{V}}+P_{i}^{\mathrm{H}}\right)
$$

where $v_{i}$ is the $\mathrm{ABS}$ speed, $P_{i}^{\mathrm{V}}$ and $P_{i}^{\mathrm{H}}$ are the power consumption for vertical and horizontal movement, respectively. Denoting the height difference between two stopping locations by $\Delta h_{i}$, the vertical and the horizontal velocities can be computed as $v_{i}^{v}=v_{i} \sin \phi_{i}$ and $v_{i}^{h}=v \sin \phi_{i}$, where $\phi_{i}=\sin ^{-1}\left(\frac{\Delta h_{i}}{D_{i}}\right)$. The horizontal power consumption $P_{i}^{H}$ is the summation of the parasitic power and the induced power for overcoming the parasitic drag. The parasitic power is given by [36]:

$P_{i}^{P}=\frac{1}{2} \rho C_{D_{o}} A_{e}\left(v_{i}^{h}\right)^{3}+\frac{\pi}{4} N_{b} c_{b} \rho C_{D_{o}} \omega_{i}^{3} R^{4}\left(1+3\left(\frac{v_{i}^{h}}{\omega_{i} R}\right)^{2}\right)$,

where $C_{D_{o}}$ is the drag coefficient, $\rho$ is the air density, $c_{b}$ is the blade chord, $N_{b}$ is the number of blades, and $A_{e}$ is the reference area. The induced power can be obtained by:

$$
P_{i}^{I}=\omega_{i} R W \mu_{i},
$$

where $R$ is the motor disk radius, $W$ is the ABS weight, and 


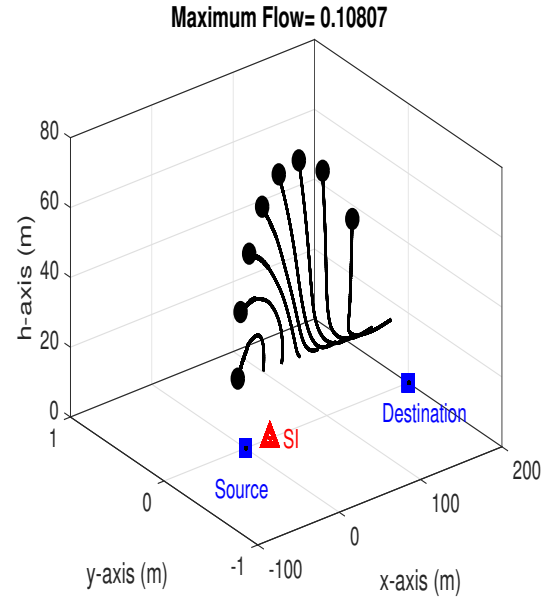

(a)

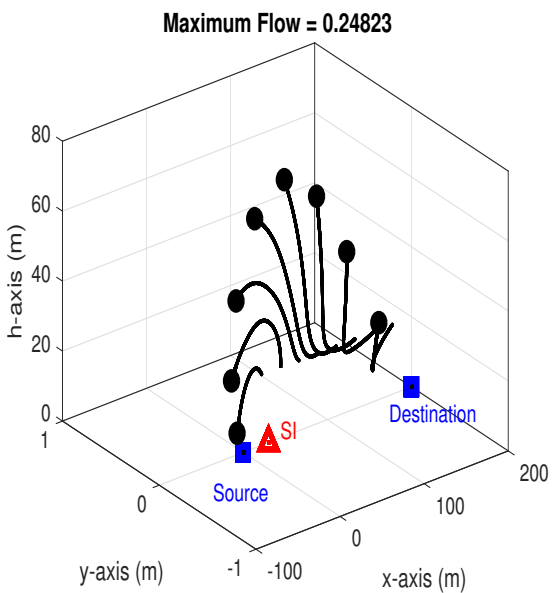

(b)

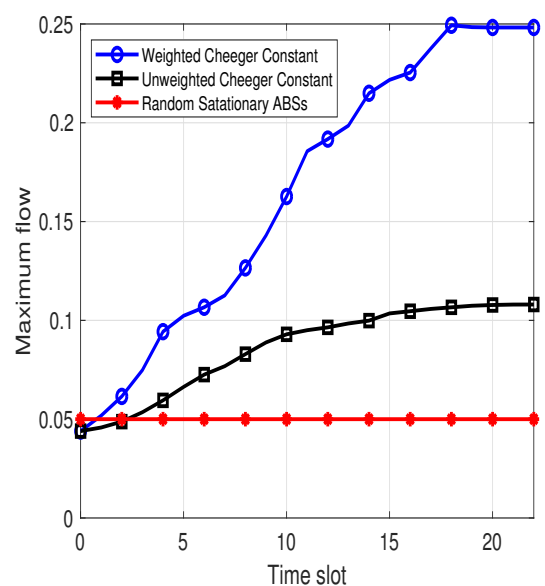

(c)

Figure 1: The trajectories of the ABSs in the presence of 1 SI based on: (a) the unweighted Cheeger constant and (b) the weighted Cheeger constant. (c) Comparison of the maximum flows throughout the network for movement of the ABSs based on the weighted/unweighted Cheeger constant and random stationary ABSs.

$\omega_{i}$ is the blade angular velocity, and $\mu_{i}$ can be obtained using:

$$
g\left(\mu_{i}\right)=2 \rho \pi \omega^{2} R^{4} \mu_{i} \sqrt{\frac{\left(v_{i}^{h}\right)^{2}}{\omega_{i}^{2} R^{2}}+\mu_{i}^{2}}-W .
$$

The power consumption for vertical movement in the climbing and the descending cases is given by [29]:

$$
P_{i}^{V}=\left\{\begin{array}{lc}
\frac{W}{2} v_{i}^{v}+\frac{W}{2} \sqrt{\left(v_{i}^{v}\right)^{2}+\frac{2 W}{\rho \pi R^{2}}}, & \text { climbing } \\
\frac{W}{2} v_{i}^{v}-\frac{W}{2} \sqrt{\left(v_{i}^{v}\right)^{2}+\frac{2 W}{\rho \pi R^{2}}}, & \text { descending. }
\end{array}\right.
$$

As can be seen from (18), the energy consumption of the ABSs is proportional to the moving time $\left(\frac{D_{i}}{v_{i}}\right), P_{i}^{V}$ and $P_{i}^{H}$. Since the ABSs should reach to their final locations at the same time, we assume equal moving times for the ABSs at each time-slot. Base on this, the energy consumption depends on the summation of $P_{i}^{V}$ and $P_{i}^{H}$ which is a non-decreasing function of the ABSs' speed. As a result, given the initial and the final locations after computing the moving directions as in (13), the most energy efficient path is the straight line connecting the two locations $q_{i}[0]$ and $q_{i}[L]$; the reason is that, considering the fixed moving time in each time-slot, the minimum distance ABS trajectory can result in the minimum speed for ABSs.

\section{Simulation Results}

We have conducted extensive simulations to evaluate the effectiveness of the proposed dynamic mobility-aware interference avoidance scheme. The parameters are presented in Table I. unless otherwise stated. In the following, we consider both single-commodity and multi-commodity maximum flow scenarios.

In the single-commodity case, a pair of source and destination aim to communicate through several ABSs in the presence of SIs from the primary network. Consider a 3D cubic space of
Table I: Simulation parameters.

\begin{tabular}{|c|c|c|}
\hline Parameter & Description & Value \\
\hline$f_{c}$ & Carrier frequency & $2 \mathrm{GHz}$ \\
\hline$B$ & Bandwidth & $1 \mathrm{~Hz}$ \\
\hline$v_{\max }$ & ABS maximum speed & $5 \mathrm{~m} / \mathrm{s}$ \\
\hline$\zeta, \kappa$ & Smoothed step function parameters & 1,10 \\
\hline$r_{\text {int }}$ & Interference radius & $5 \mathrm{~m}$ \\
\hline$\mu_{\mathrm{LoS}}, \mu_{\mathrm{NLoS}}$ & Attenuation factors for LoS/NLoS & $5 \mathrm{~dB}, 20 \mathrm{~dB}$ \\
\hline$\psi, \eta$ & Constant values for $P_{i, j}^{\mathrm{LoS}}$ & $11.95,0.14$ \\
\hline$\rho$ & Air density & $1.225 \mathrm{~kg} / \mathrm{m}^{-3}$ \\
\hline$\omega$ & Angular velocity & $20 \mathrm{rad} / \mathrm{sec}$ \\
\hline$R$ & Rotor disk radius & $0.5 \mathrm{~m}$ \\
\hline$c_{b}$ & Blade chord & $10 \mathrm{~cm}$ \\
\hline$N_{b}$ & Number of blades & 4 \\
\hline$W$ & Weight of ABS & $50 \mathrm{~N}$ \\
\hline
\end{tabular}

size $200 \mathrm{~m} \times 200 \mathrm{~m} \times 100 \mathrm{~m}$, in which 8 ABSs are deployed. Each ABS $i \in\{1,2, \ldots, 8\}$ is initially located at $(0,25 i, 20)$ $\mathrm{m}$. That is, the ABSs are in a straight line at the height of 20 meters from the ground with equal distances of $25 \mathrm{~m}$ in the $x$ coordinate. The source and the destination are fixed on the ground at positions $(0,0,0) \mathrm{m}$ and $(200,0,0) \mathrm{m}$. The moving directions of the ABSs are computed using weighted and unweighted Cheeger constants. We use random stationary ABS positioning as a baseline for comparison. In this baseline, the ABSs are randomly positioned on a $200 \mathrm{~m} \times 200 \mathrm{~m}$ plane with a fixed height of $20 \mathrm{~m}$. All the simulation results are averaged over 1000 Monte Carlo runs.

As seen in Fig. 1(a) and Fig. 1(b), one SI is located at $(30,0,0) \mathrm{m}$. The ABSs are moving in a 3D space. The black circles and black lines represent the ABSs and their trajectories towards maximum flow locations, respectively. As seen in Fig. 1(c), the mobility of the ABSs can considerably increase 


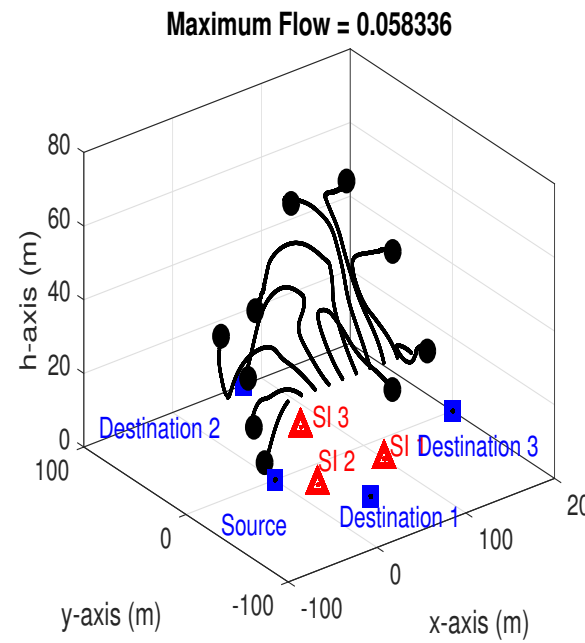

(a)

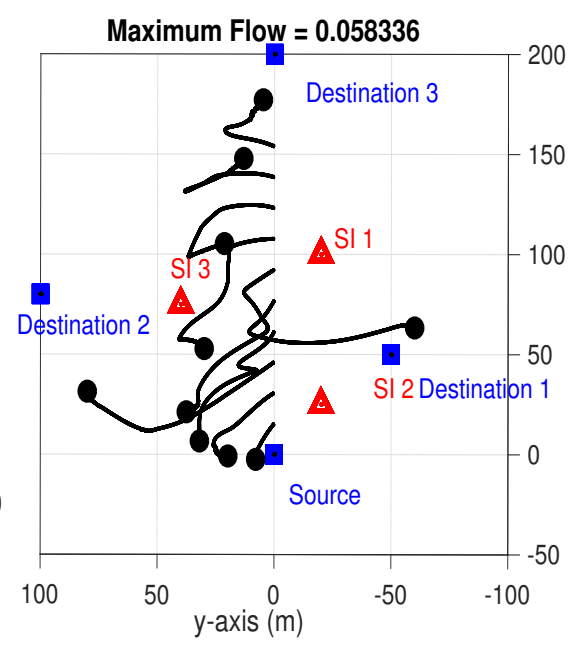

(b)

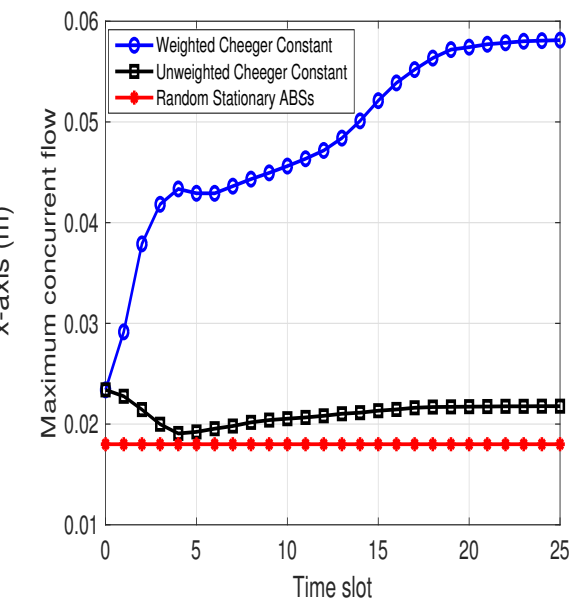

(c)

Figure 2: The trajectories of the ABSs in the presence of 3 SIs using the weighted Cheeger constant for a multi-cast scenario: (a) 3D view, (b) Top view. (c) Comparison of the maximum flow throughout the network for movement of the ABSs based on the weighted/unweighted Cheeger constant and random stationary ABSs.

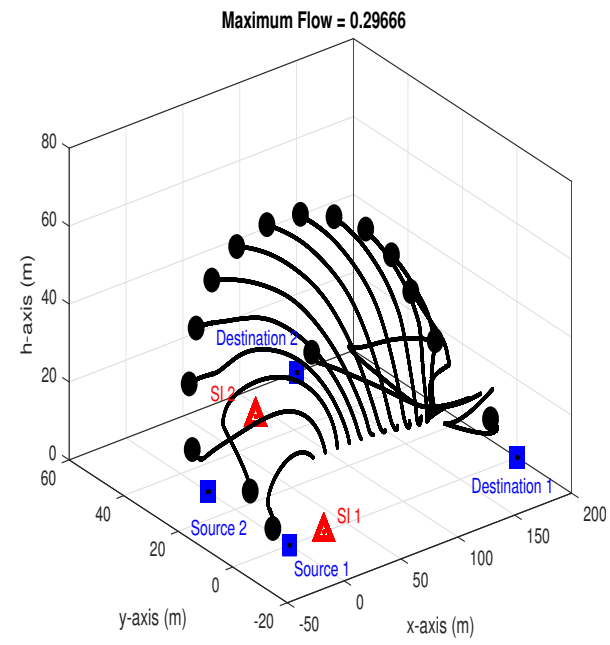

(a)

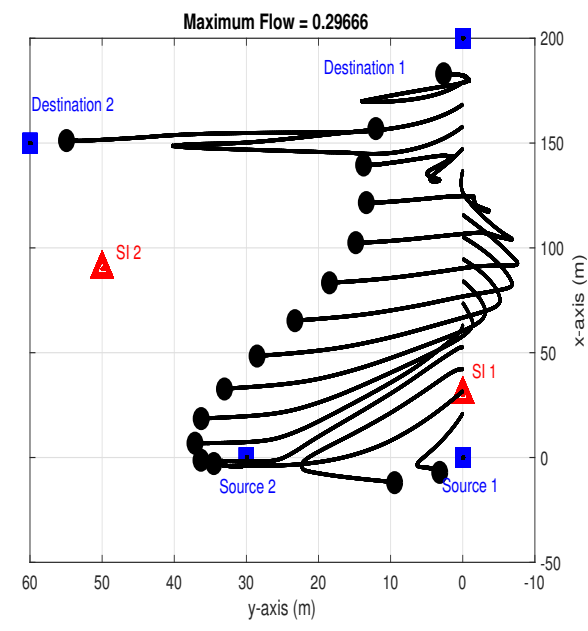

(b)

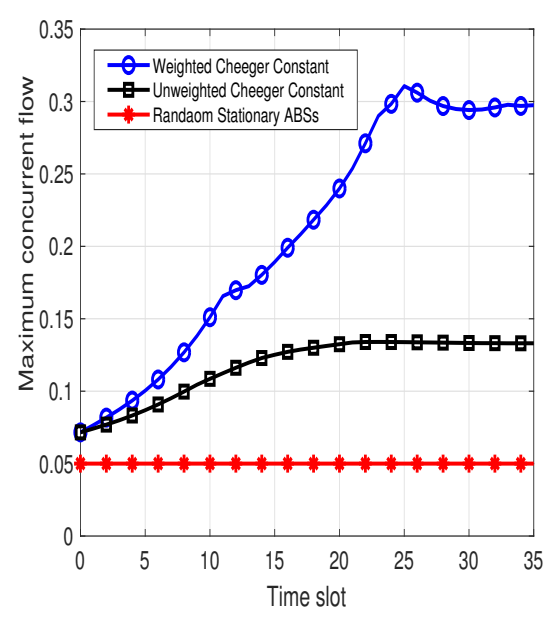

(c)

Figure 3: The trajectories of the ABSs in the presence of 2 SIs using the weighted Cheeger constant for a multi-unicast scenario: (a) 3D view, (b) Top view. (c) Comparison of the maximum flow throughout the network for movement of the ABSs based on the weighted/unweighted Cheeger constant and random stationary ABSs.

the maximum flow as compared to stationary deployment. Moreover, the weighted Cheeger constant further improves the maximum flow by about $150 \%$ as compared to the conventional Cheeger constant.

In Fig. 2, a multi-cast scenario is considered in which one source aims at transmitting data to three destinations in the presence of 3 SIs. Here, 12 ABSs are considered. Fig. 2(a) shows the $3 \mathrm{D}$ view of the ABS trajectories, while the top view in Fig. 2(b) can help better illustrate the final locations. Fig. 2(c) shows the effectiveness of the weighted Cheeger constant in comparison to the other two baselines.
In Fig. 3, a multi-unicast case is considered in which two pairs of source and destination aim to communicate. Here, 16 ABSs are deployed. The 3D view in Fig. 3(a) and the top view in Fig. 3(b) present the positions of the ABSs towards the maximum flow. In Fig. 3(b), it can be seen that one of the ABSs is changing its direction toward destination 2 to form a better link for the data flow. As shown in Fig. 3(c), the weighted Cheeger constant outperforms the other two approaches.

Considering the single-commodity case scenario, in Fig. 4 the maximum flows of the network are compared for the cases of centralized and distributed computation of the Feidler 

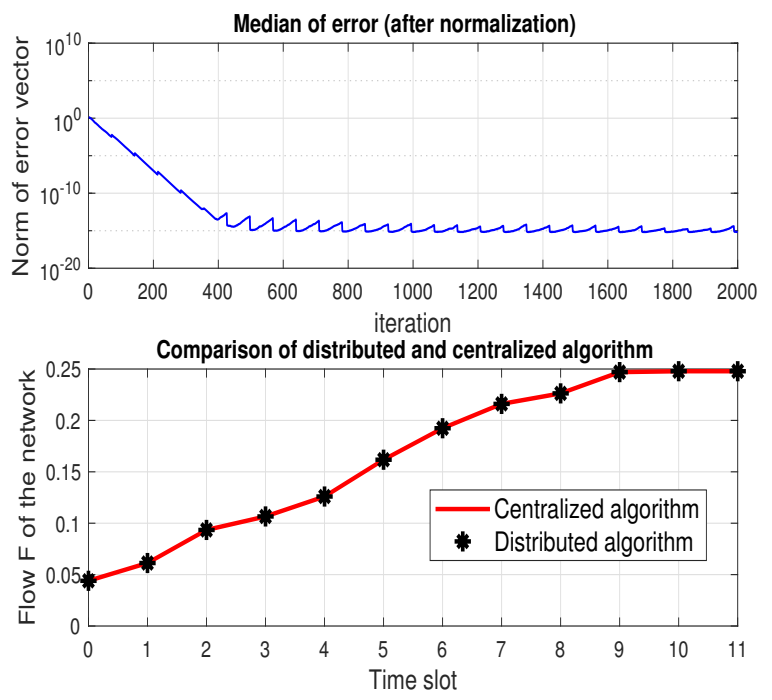

Figure 4: Comparison of the distributed algorithm and the centralized algorithm for the computation of the Fiedler vector on the network maximum flow while deploying the weighted Cheeger constant.

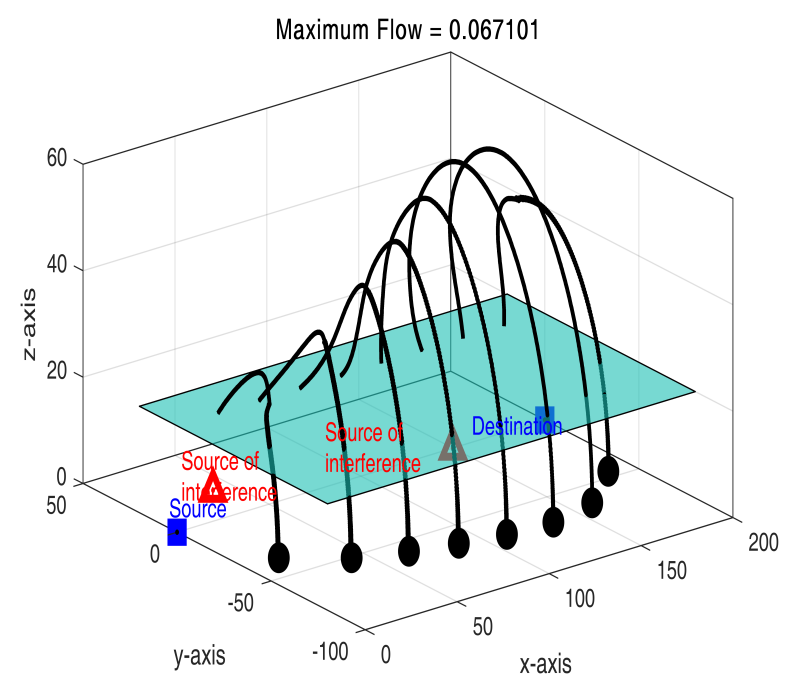

Figure 5: Depending on the SI positions, in some scenarios, the ABSs move towards the positions with zero height.

vector. In the top sub-figure, the norm of the error vector for the Fiedler vector is computed over the iterations for the distributed algorithm. As can be seen, if the number of iterations is sufficiently large, the error of the distributed algorithm is negligible. Hence, the results obtained form the distributed algorithm and the centralized algorithm are identical, as shown in the bottom sub-figure.

In simulations, if no constraints are imposed for the heights of the ABSs, it can be seen that, in some situations, the final heights of the ABSs are equal to zero, which is impractical. Such a case may occur when there is no SI on one side of the region of interest. To address this issue, we may impose a
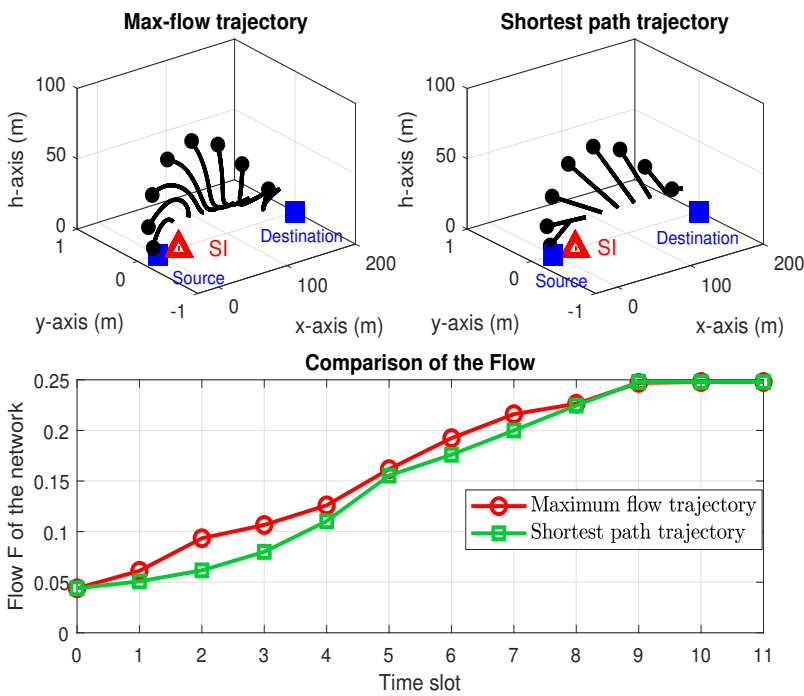

Figure 6: Comparison of the network flows for maximum flow trajectory versus shortest path trajectory in a single-commodity scenario.

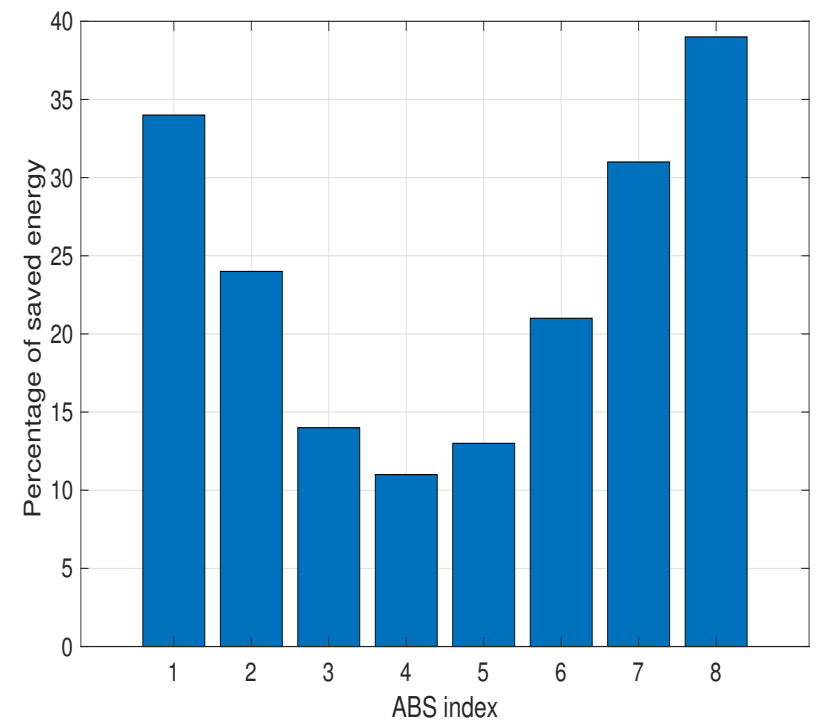

Figure 7: Percentage of the saved energy for different trajectories schemes for each ABS.

height constraint for each ABS as the green plane shown in Fig. 5 to prevent the ABSs from moving to an amplitude lower than $h=20 \mathrm{~m}$. In the simulated scenario, this leads to a loss of 0.023 of maximum flow for the given source-destination pair.

In Fig. 6, the flows of the network with the maximum flow trajectory (obtained by using the methods in Section IV-C) and the energy-efficient trajectory (obtained by using the method in Section VI-C) are compared. The percentages of saved energy for different ABSs are compared in Fig. 7, which is defined as $\epsilon_{i}=100 \times\left(1-E_{i}^{\text {Energy-efficient }} / E_{i}^{\text {Maximum-flow }}\right)$. For this simulation setting, the single-commodity maximum flow problem is considered in the presence of one SI. The 3D plots 
for different trajectories are included in Fig. 6 . As can be seen from Fig. 6, the loss in flow of the network is temporary and insignificant. On the other hand, as shown in Fig. 7, each ABS can save energy from about $11 \%$ to $39 \%$, depending on its initial location. One important observation is that for the ABSs that are in the middle during the initial deployment, the ratio of the saved energy is lower since their trajectories are closer to the shortest path.

\section{CONCLUSION}

In this paper, an adaptive mobility aware interference avoidance scheme is considered for ABSs in which the ABSs aim at forming a temporary network to send information from a terrestrial source to a terrestrial destination in coexistence with a primary network. The primary network causes interference to the ABS temporary network. Due to the inherent feature of the ABSs, i.e., their mobility, they can reconfigure their locations to avoid the interference. Using the weighted Cheeger constant as the connectivity metric, the optimal moving directions to achieve the maximum flow is obtained for the ABSs. Furthermore, a distributed algorithm for computing the moving directions is proposed, and the trade-off between maximum flow and energy efficient trajectories is investigated. The simulation results confirm the effectiveness of the proposed scheme, in which each ABS can save up to $39 \%$ of the energy with marginal degradation in maximum flow.

\section{REFERENCES}

[1] S. Hayat, E. Yanmaz, and R. Muzaffar, "Survey on unmanned aerial vehicle networks for civil applications: A communications viewpoint.,' Commun. Surveys Tuts., vol. 18, no. 4, pp. 2624-2661, 2016.

[2] L. Gupta, R. Jain, and G. Vaszkun, "Survey of important issues in UAV communication networks," IEEE Commun. Surveys Tuts., vol. 18, no. 2 pp. 1123-1152, 2015.

[3] M. Mozaffari, W. Saad, M. Bennis, Y.-H. Nam, and M. Debbah, "A tutorial on UAVs for wireless networks: Applications, challenges, and open problems," arXiv preprint arXiv:1803.00680, 2018.

[4] I. Parvez, A. Rahmati, I. Guvenc, A. I. Sarwat, and H. Dai, "A survey on low latency towards 5G: RAN, core network and caching solutions,' IEEE Commun. Surveys Tuts., vol. 20, pp. 3098-3130, 4th-quarter 2018

[5] M. Tavana, A. Rahmati, and V. Shah-Mansouri, "Congestion control with adaptive access class barring for LTE M2M overload using kalman filters," Computer Networks, 2018.

[6] C. Stöcker, R. Bennett, F. Nex, M. Gerke, and J. Zevenbergen, "Review of the current state of UAV regulations," Remote sensing, vol. 9, no. 5, p. 459, 2017.

[7] Y. Saleem, M. H. Rehmani, and S. Zeadally, "Integration of cognitive radio technology with unmanned aerial vehicles: issues, opportunities, and future research challenges," Journal of Network and Computer Applications, vol. 50, pp. 15-31, 2015.

[8] L. Sboui, H. Ghazzai, Z. Rezki, and M. S. Alouini, "Dynamic spectrum management in green cognitive radio cellular networks," in Proc. of IEEE International Symposium on Personal, Indoor, and Mobile Radio Communications (PIMRC), pp. 1-6, Montreal, QC, Canada, Oct 2017.

[9] D. Athukoralage, I. Guvenc, W. Saad, and M. Bennis, "Regret based learning for UAV assisted LTE-U/WiFi public safety networks," in Proc. of IEEE Global Communication Conf. (GLOBECOM), pp. 1-7, Washington, DC, USA, Dec. 2016.

[10] Y. Zeng and R. Zhang, "Energy-efficient UAV communication with trajectory optimization," IEEE Trans. Wireless Commun., vol. 16, no. 6 , pp. 3747-3760, 2017.

[11] M. Mozaffari, A. T. Z. Kasgari, W. Saad, M. Bennis, and M. Debbah, "Beyond 5G with UAVs: Foundations of a 3D wireless cellular network," arXiv preprint arXiv:1805.06532, 2018.
[12] S. Rohde and C. Wietfeld, "Interference aware positioning of aerial relays for cell overload and outage compensation," in Proc. IEEE Vehic Technol. Conf. (VTC), pp. 1-5, Quebec City, QC, Canada, Dec. 2012.

[13] U. Challita, A. Ferdowsi, M. Chen, and W. Saad, "Artificial intelligence for wireless connectivity and security of cellular-connected UAVs," arXiv preprint arXiv:1804.05348, 2018.

[14] M. Abdel-Malek, A. S. Ibrahim, and M. Mokhtar, "Optimum UAV positioning for better coverage-connectivity tradeoff," in Proc. of IEEE Int. Symp. Personal, Indoor, and Mobile Radio Commun. (PIMRC), Montreal, QC, Canada , Oct 2017.

[15] Z. Han, A. L. Swindlehurst, and K. R. Liu, "Optimization of MANET connectivity via smart deployment/movement of unmanned air vehicles,' IEEE Trans. Vehic. Technol., vol. 58, no. 7, pp. 3533-3546, 2009.

[16] M. U. Chowdhury, S. J. Maeng, I. Guvenc, and E. Bulut, "Effects of 3D antenna radiation and two-hop relaying on optimal UAV trajectory in cellular networks," in Proc. IEEE Aerospace Conf., Big Sky, ID, Mar., 2019.

[17] E. Bulut and I. Guvenc, "Trajectory optimization for cellular-connected UAVs with disconnectivity constraint," in Proc. IEEE Int. Conf. Commun. (ICC) Workshops, Kansas City, MO, May 2018.

[18] H. Nagarajan, S. Rathinam, and S. Darbha, "On maximizing algebraic connectivity of networks for various engineering applications," in Proc. of European Control Conf. (ECC), pp. 1626-1632, July Linz, Austria, Nov. 2015.

[19] H. Nagarajan, S. Rathinam, S. Darbha, and K. R. Rajagopal, "Synthesizing robust communication networks for UAVs," in Proc. IEEE American Control Conference (ACC), pp. 3730-3735, Montreal, QC, Canada, June 2012.

[20] A. Rahmati and V. Shah-Mansouri, "Price-based power control in relay networks using stackelberg game," in IEEE 23rd Iranian Conf. on Elect. Eng. (ICEE),, pp. 263-267, 2015.

[21] Q. Wu, Y. Zeng, and R. Zhang, "Joint trajectory and communication design for multi-uav enabled wireless networks," IEEE Trans. Wireless Commun., vol. 17, no. 3, pp. 2109-2121, 2018.

[22] M. R. Javan, N. Mokari, F. Alavi, and A. Rahmati, "Resource allocation in decode-and-forward cooperative communication networks with limited rate feedback channel," IEEE Trans. Vehic. Technol., vol. 66, no. 1, pp. 256-267, 2017.

[23] P. Demestichas, A. Georgakopoulos, D. Karvounas, K. Tsagkaris, V. Stavroulaki, J. Lu, C. Xiong, and J. Yao, "5G on the horizon: Key challenges for the radio-access network," IEEE vehic. technol. mag., vol. 8, no. 3, pp. 47-53, 2013.

[24] B. Khamidehi, A. Rahmati, and M. Sabbaghian, "Joint sub-channel assignment and power allocation in heterogeneous networks: An efficient optimization method," IEEE Commun. Lett., vol. 20, no. 12, pp. 2490 2493, 2016

[25] M. Tavana, A. Rahmati, V. Shah-Mansouri, and B. Maham, "Cooperative sensing with joint energy and correlation detection in cognitive radio networks," IEEE Commun. Lett., vol. 21, no. 1, pp. 132-135, 2017.

[26] F. R. Chung and F. C. Graham, Spectral graph theory. No. 92, American Mathematical Soc., 1997.

[27] U. Challita and W. Saad, "Network formation in the sky: Unmanned aerial vehicles for multi-hop wireless backhauling," in in Proc. IEEE GLOBECOM, pp. 1-6, Singapore, Dec 2017.

[28] A. Al-Hourani, S. Kandeepan, and A. Jamalipour, "Modeling air-toground path loss for low altitude platforms in urban environments," in Proc. of IEEE Global Commun. Conf. (GLOBECOM), pp. 2898-2904, Austin, TX, USA, Dec. 2014.

[29] M. Mozaffari, W. Saad, M. Bennis, and M. Debbah, "Mobile unmanned aerial vehicles (UAVs) for energy-efficient internet of things communications," IEEE Trans. Wireless Commun., vol. 16, no. 11, pp. 7574-7589, 2017.

[30] L. R. Ford and D. R. Fulkerson, "Maximal flow through a network," Canadian journal of Mathematics, vol. 8, no. 3, pp. 399-404, 1956.

[31] N. M. M. De Abreu, "Old and new results on algebraic connectivity of graphs," Linear algebra and its applications, vol. 423, no. 1, pp. 53-73, 2007.

[32] X. He, H. Dai, and P. Ning, "Dynamic adaptive anti-jamming via controlled mobility," IEEE Trans. Wireless Commun., vol. 13, pp. 4374 4388, Aug 2014.

[33] T. Leighton and S. Rao, "Multicommodity max-flow min-cut theorems and their use in designing approximation algorithms," Journal of the ACM (JACM), vol. 46, no. 6, pp. 787-832, 1999. 
[34] A. Bertrand and M. Moonen, "Distributed computation of the fiedler vector with application to topology inference in ad hoc networks," Signal Processing, vol. 93, no. 5, pp. 1106-1117, 2013.

[35] C. Di Franco and G. Buttazzo, "Energy-aware coverage path planning of UAVs," in Proc. IEEE Int. Conf. Autonomous Robot Systems and Competitions (ICARSC), pp. 111-117, Vila Real, Portugal, April 2015.

[36] Y. Zeng and R. Zhang, "Energy-efficient UAV communication with trajectory optimization," IEEE Trans. on Wireless Commun., vol. 16, pp. 3747-3760, 2017, June 2017. 